\title{
Numerical Modeling for Atomization of Coaxial Liquid/Gas Jets
}

\section{Stephen Gen-Ken Chuech}

Associate professor, Department of Mechanical and Mechatronic Engineering, National Taiwan Ocean University, Keelung, Taiwan 20224, sgc@mail.ntou.edu.tw

Andrzej J. Przekwas

Manager of Research Projects, CFD Research Corporation, Huntsville, Alabama 35805, USA.

Chih-Yuan Wang

Graduate Student, Department of Mechanical and Mechatronic Engineering, National Taiwan Ocean University, Keelung, Taiwan 20224.

Follow this and additional works at: https://jmstt.ntou.edu.tw/journal

Part of the Mechanical Engineering Commons

\section{Recommended Citation}

Chuech, Stephen Gen-Ken; Przekwas, Andrzej J.; and Wang, Chih-Yuan (2004) "Numerical Modeling for Atomization of Coaxial Liquid/Gas Jets," Journal of Marine Science and Technology. Vol. 12: Iss. 4, Article 8.

DOI: 10.51400/2709-6998.2249

Available at: https://jmstt.ntou.edu.tw/journal/vol12/iss4/8

This Research Article is brought to you for free and open access by Journal of Marine Science and Technology. It has been accepted for inclusion in Journal of Marine Science and Technology by an authorized editor of Journal of Marine Science and Technology. 


\title{
NUMERICAL MODELING FOR ATOMIZATION OF COAXIAL LIQUID/GAS JETS
}

\author{
Stephen Gen-Ken Chuech*, Andrzej J. Przekwas**, and Chih-Yuan Wang***
}

Key words: numerical, atomization, coaxial, jet.

\begin{abstract}
In the present study, the atomization of a coaxial injector in the liquid/gas engine combustor was numerically investigated. Based on the wave instability analysis of the interface between liquid and gas, an atomization model for the high-speed liquid jets was established by coupling with the jet flow solutions. First, the wave instability was analyzed to predict the growth rates along the liquid jet surface by numerically solving the wave dispersion equation, while the governing equations of the liquid flow were solved for the formation of the liquid jet core and the variation of the liquid flow structure. Then, these two solutions were coupled in the atomization model to predict drop size distribution and breakup rate. In the present study, the coaxial injection combustion model (CICM) code, which was based on the experimental data for a coaxial injector in the space shuttle main engine (SSME), was used to verify the coupled model. The LOX/GH2 coaxial injector of the SSME preburner was considered as the verification case. The present atomization analyses for the case are in good agreement with the results of the CICM code.
\end{abstract}

\section{INTRODUCTION}

The liquid jet atomization has been widely utilized for many industrial applications, such as: gas turbine combustors, rocket thrust chambers, and diesel engines. In the combustion chamber, drop breakup rate, drop size and velocity distribution, and intact jet-core length have profound influences on the combustion efficiency and emission pollution. Despite a great quantity of past studies $[10,22]$, the physical process of atomization phenomenon has not been fully understood. Especially, most of these studies were focused on the experimental work and limited to the atomization of pressure and

Paper Submitted 06/04/04, Accepted 09/24/04. Author for Correspondence: Stephen G. Chuech. E-mail: sgc@mail.ntou.edu.tw.

*Associate professor, Department of Mechanical and Mechatronic Engineering, National Taiwan Ocean University, Keelung, Taiwan 20224.

**Manager of Research Projects, CFD Research Corporation, Huntsville, Alabama 35805, USA.

***Graduate Student, Department of Mechanical and Mechatronic Engineering, National Taiwan Ocean University, Keelung, Taiwan 20224. airblast types. The liquid/gas coaxial nozzle was received relatively less attention.

Most of the liquid rocket combustors use a large number of coaxial injectors. For example, in the space shuttle main engine power head, each of the three combustors: fuel-side and oxidizer-side preburners and the main combustion chamber, has over 200 injectors. The injector design is of vital importance to the chamber performance. Figure 1 shows the schematic of a single coaxial injector of the SSME preburner. Beginning at the injector exit, a series of complex physico-chemical processes occur, which result in the large heat release rate within the rocket combustor, as shown in Figure 2. These complex processes, including: liquid jet atomization, liquid evaporation, turbulent mixing, and chemical reaction, are still not well understood. Among them, the atomization has the most profound influence on the designed combustor size, combustion efficiency and stability. However, atomization is also the least understood phenomena in the spray combustion process.

Past investigations of dense sprays were primarily limited to analyses based on observations near the boundaries of dilute dispersed jets [5]. Detailed reviews of these experimental observations and theoretical analy-

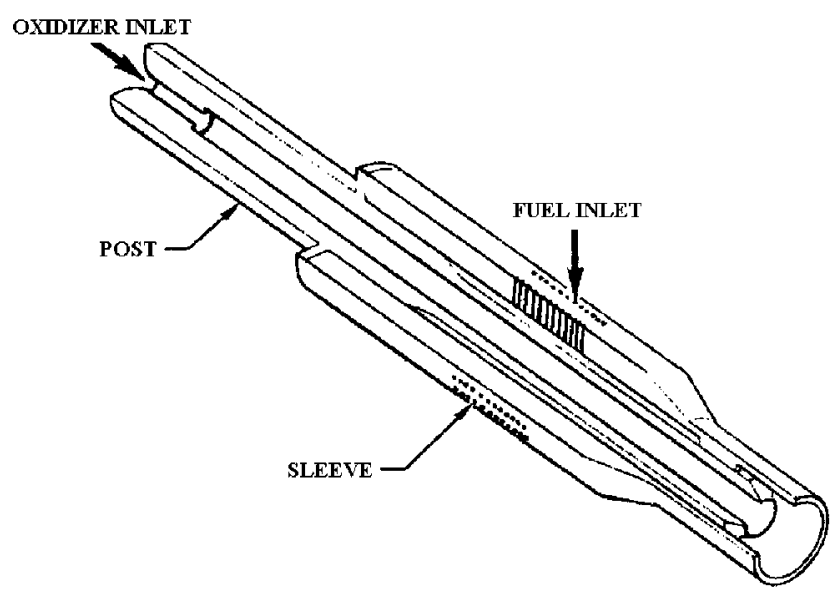

Fig. 1. A single coaxial injector element of the space shuttle main engine. 
ses of dense sprays have been provided by Faeth [5], Lefebvre [11], Sirignano [23], Reitz and Bracco [20], and Lin and Reitz [15]. However, the atomization conditions due to the primary breakup from the liquid jet surface have not been well defined. In the 1960's, Mayer [16] and Adelberg [1,2] predicted mean droplet size and mean breakup rate on primary atomization by using the work of Lamb [9] and Jeffreys [8]. Since then, Mayer's model [16] has been used as a standard method for the evaluation of the liquid propellant rocket engines. It seems that the atomization model is the weakest element of the current JANNAF Standard Prediction Procedure [25]. The model is limited to coaxial elements and still uses the semi-empirical formulae to compute the liquid jet stripping rate and breakup droplet diameters. The empirical constants for the model were obtained for specific injection conditions and were not consistent with the hot fire engine data. The extension of applicability of the model, for the modern combustion chambers, is questionable. Recently, several experimental studies $[3,17,18]$ on the coaxial liquid/gas atomization have led to a more profound understanding of the processes of injection, mixing, and combustion in a rocket engine. Nevertheless, existing atomization models for liquid rocket engines still rely on empirical formulae to predict the breakup rates and drop sizes, which have a very narrow range of applicability. Therefore, better theoretical modeling for the atomization process, based on the first principles, is necessary and crucial for the development of advanced liquid propellant combustion systems.

Regarding the modeling of jet atomization, there were three types of direct numerical simulations for high-speed liquid injection, namely the volume of fluid (VOF) technique [6], the jet embedding (JE) technique $[4,19]$, and the blob-injection (BI) method [21]. Liang $[13,14]$ applied the VOF technique of Hirt and Nichols

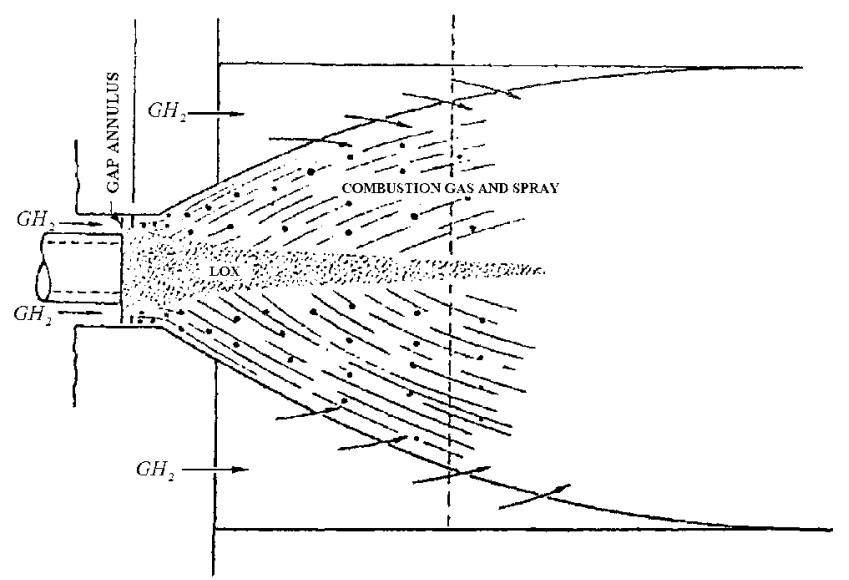

Fig. 2. Schematics of flow structure in the vicinity of the coaxial injector.
[6] to predict atomization of a single axisymmetric liquid jet. In the analysis, two sets of momentum and continuity equations were solved over the entire computational domain. It can resolve only the overall jet shape and will not capture the wave formation and growth on the jet surface. The major limitation of the VOF technique is that it requires very fine grid distribution within the liquid jet and its gaseous neighborhood even for predicting the long wavelength waves. Therefore, its use for practical rocket injection systems with hundreds of injector elements is doubtful. The JE technique [19] is an alternative to the VOF technique for this class of problems. It utilizes the multi-grid concept by employing the regular grid for the gas phase, and the embedded, adaptive grid for the liquid jet core. As a result, relative to VOF, it is economical and suitable for both single and multi-injector flow analyses. Later, Reitz [21] developed the BI method for analyzing the fuel atomization of pressure atomizers in diesel engines. The method, assuming instantaneous blob formation at the injection exit, is not capable of representing the liquid jet core. Also, its use of linearized theory to compute the rate of blob shattering may have limited applications for turbulent or high-speed flows. It can, however, prove a viable tool for simulating the secondary breakup and droplet coagulation by using the population evolution theory (i.e., the 'parent-child' approach).

Although the JE and VOF techniques can represent the liquid jet core, they are unable to directly predict primary breakup rates and drop sizes. Both of the methods have to employ empirical correlations for these quantities to simulate the liquid injection. Among them, only the numerical work of Chuech et al. [4] was aimed at elimination of the need for such empirical input by developing an atomization model from the first principles. However, the assumed constant gas velocity along the liquid/gas interface, used in the reported model, does not adequately represent the real flow pattern in the vicinity of the injector. Therefore, in the present study, the JE method with the variation in gas phase motion was adopted to couple with surface wave dynamics analysis for the atomization model. The objectives of the modeling included the formulation and solutions of the model for the liquid jet flow structure and the wave growth along the liquid/gas interface. The model is also validated by comparisons with the CICM calibrated data for $\mathrm{LOX} / \mathrm{GH}_{2}$ flow experiments [25] and the VOF analysis [14]. Further details are discussed in following sections.

\section{ATOMIZATION MODELING}

Advantages and limitations of the methods for simulating the coaxial liquid/gas injection and atomiza- 
tion were briefly stated in the previous section. Among these methods, the JE technique has been chosen for the present study. This is because its economic adaptive grid system can capture the formation and growth of short waves, which are important for primary breakup on the liquid jet surface. In addition, the JE technique has potential for future application to non-axisymmetric jets by representing the jet shape in the elliptical form rather than in the round one. In the modeling, the major task in the present study was to couple the wave dynamics analysis with the JE solutions for predicting liquid breakup rates and drop sizes instead of using empirical correlations for the mass-stripping rate on the jet surface. The mathematical formulation for the liquid flow motion was first reported as follows:

\section{Basic equations of liquid flow}

The continuity and momentum equations in conservation form for liquid jet flow motion with mass transfer from the jet surface can be expressed as:

$$
\begin{aligned}
& \partial_{t} \rho+\nabla \cdot(\rho V)=S_{c} \\
& \partial_{t}\left(\rho v_{j}\right)+\nabla \cdot\left(\rho v_{j} V\right)=-\partial_{j}(p)+\nabla \cdot \tau_{i j}+S_{m}
\end{aligned}
$$

where $S_{c}$ and $S_{m}$ represent the source terms due to atomized effects and the subscript $j$ denotes the coordinate in each direction. In the present study, with the assumption of the one-dimensional steady-state isobaric flow, the system of Eqs. (1) and (2) based on an adaptive surface-confirming grid along the jet path $\xi$ can be further rewritten as:

$$
\begin{aligned}
& \partial_{\xi}(\rho u)=-\dot{m}_{B}^{\prime} \\
& \partial_{\xi}\left(\rho u^{2}\right)=-\dot{m}_{B}^{\prime}\left(u-u_{d}\right)-\partial_{\xi} \varepsilon
\end{aligned}
$$

The friction gain or loss to liquid is depended upon the sign of the friction $\varepsilon$ between the liquid and gas at the jet interface. The friction $\varepsilon$ may be assumed as:

$$
\varepsilon=\frac{C_{f} \cdot \rho\left(u-u_{g}\right) \cdot\left|u-u_{g}\right|}{2}
$$

where

$$
C_{f}=0.024 R e^{-0.2}
$$

Note that $C_{f}$ is a frictional coefficient for the liquid cylinder in the coaxial flow direction at turbulent conditions [19], even though only the mean flow is considered for the present model.

The above nonlinear equations are numerically integrated along the jet path and then linearized by an implicit, fully conservative Newton-Raphson method. As a result, two algebraic equations can be solved for the liquid jet radius $r$ and axial velocity $u$ simultaneously at each jet cross-section. The Newton-Raphson approach guarantees a second order convergence, which only requires two to three iterations at each integration point. Note that the local breakup rate $\dot{m}_{B}$ can be computed through the surface wave instability analysis in the present study, whereas it has been estimated from the empirical formulae in the past computations $[13,14$, 19].

\section{Surface wave dynamics}

As a liquid jet issues from a nozzle, its surface is always subjected to disturbances, including vibrations of the nozzle, gas motion surrounding the jet, turbulence in the liquid, and roughness of the nozzle surface. For linear temporal stability analysis, the growth rate $\omega$ of the disturbance can be described in the wave dispersion equation [12]:

$$
\begin{gathered}
\omega^{2}+2 v n^{2}\left[\frac{I_{1}^{\prime}(n r)}{I_{0}(n r)}-\frac{2 n l}{n^{2}+l^{2}} \cdot \frac{I_{1}(n r)}{I_{0}(n r)} \cdot \frac{I_{1}^{\prime}(1 r)}{I_{1}(1 r)}\right] \cdot \omega \\
=\frac{\sigma \cdot n}{\rho r^{2}}\left(1-n^{2} r^{2}\right)\left(\frac{1^{2}-n^{2}}{1^{2}+n^{2}}\right) \cdot \frac{I_{1}(n r)}{I_{0}(n r)} \\
+\frac{\rho_{g} u_{R}^{2} \cdot n^{2}}{\rho}\left(\frac{1^{2}-n^{2}}{1^{2}+n^{2}}\right) \cdot \frac{I_{1}(n r)}{I_{0}(n r)} \cdot \frac{K_{0}(n r)}{K_{1}(n r)}
\end{gathered}
$$

where the modified wavenumber $1=\sqrt{n^{2}+\omega / v}$. The previous study [4] examined the dispersion equation in full form, and showed that the limited solutions of the simplified form can still provide considerably useful information. Therefore, two limited cases, namely $n r$ > 1 for the short wavelength region and $n r<<1$ for the long wavelength region, were considered in the wave instability analysis of the present study. For high-speed liquid jets with long-wavelength disturbances, the following approximation to the ratio of the Bessel functions can be applied [26]:

$$
\begin{aligned}
& I_{0}(n r) \approx 1 \\
& I_{0}^{\prime}(n r) \approx \frac{n r}{2} \\
& I_{1}(n r) \approx \frac{n r}{2} \text { and } I_{1}(1 r) \approx \frac{1 r}{2} \\
& I_{1}^{\prime}(n r) \approx I_{1}^{\prime}(1 r) \approx \frac{1}{2}
\end{aligned}
$$




$$
\frac{K_{0}(n r)}{K_{1}(n r)} \approx-n r \cdot \ln \left(\frac{n r}{2}\right)
$$

With the inequality of $1^{2}>n^{2}$ [12], the dispersion equation can be simplified as:

$$
\omega^{2}+v n^{2} \omega=\frac{\sigma n^{2}\left(1-n^{2} r^{2}\right)}{2 \rho r}-\frac{\rho_{g} r^{2} n^{4} u_{R}^{2}}{2 \rho} \cdot \ln \left(\frac{n r}{2}\right)
$$

In the case of short waves, the Bessel functions are approximated as follows:

$$
\begin{aligned}
& I_{1}(n r) \approx I_{0}(n r) \approx \frac{e^{n r}}{\sqrt{2 \lambda n r}} \\
& I_{1}(n r) \approx I_{0}(n r) \approx \frac{e^{n r}}{\sqrt{2 \pi n r}} \\
& I_{1}(1 r) \approx I_{0}(1 r) \approx \frac{e^{1 r}}{\sqrt{2 \pi 1 r}} \\
& \frac{I_{1}^{\prime}(n r)}{I_{0}(n r)} \approx \frac{I_{1}^{\prime}(1 r)}{I_{1}(1 r)} \approx 1 \\
& K_{0}(n r) \approx K_{1}(n r) \approx \sqrt{\frac{\pi}{2 n r}} e^{-n r}
\end{aligned}
$$

Simplifying Eq. (7), the approximate dispersion equation for the short-wavelength disturbance becomes:

$$
\omega^{2}+2 v n^{2} \omega=-\frac{\sigma n^{3}}{\rho}+\frac{\rho_{g} n^{2} u_{R}^{2}}{\rho}
$$

\section{Formation of liquid droplet}

By imposing an infinitesimal axisymmetric disturbance with a spectrum of wavelengths, the dispersion equation, Eqs. (13) and (19) determines the growth rates for the amplitudes of all waves. The wave growth is expressed in Fourier form [24]:

$$
\eta=\eta_{0} e^{i n \xi+\omega t}
$$

If the real part of the solution $\omega$ in Eq. (13) and (19) is greater than zero (i.e., $\omega_{r}>0$ ), the exponential wave growth occurs; if it is negative, wave damping takes place. In the present model, the ligaments or drops are assumed to separate from the liquid jet surface due to the growth of initial disturbances above critical levels. The criterion for ligaments stripping from the growing waves is based on the assumption that when the amplitude of the growing wave $\eta_{i}$ is larger than its own wavelength $\lambda_{i}$, the crest of the wave is erupted as a ligament. This criterion can be expressed in the following form:

$$
\eta_{0} e^{\left(\omega_{r}\right)_{i} \cdot\left(t_{B}\right)_{i}} \geq \lambda_{i}, i=1,2,3, \ldots, \infty
$$

The separated ligament, in the form of pulsating ring of cross-section diameter equal to $\lambda_{i} / 2$, breaks into a number of drops of similar size. With this assumption, the drop size is equal to $\lambda_{i} / 2$ but does not necessarily correspond to the fastest growing wavelength, since it is possible for some shorter waves, with less growth rates, to take a shorter amount of time to grow up to their own critical wavelengths. After the first drops have been detached, this stripping action must leave smaller ripples on the liquid surface. Therefore, the breakup of other waves of shorter wavelength than $\lambda_{i}$ can consequently occur as long as their growth rates $\left(\omega_{r}\right)_{i}$ are positive. Accordingly, the breakup time $\left(t_{B}\right)_{i}$ needed for each qualified wave to grow to the breakup level can be computed through Eq. (21). The volume of the ring ligament can also be obtained:

$$
\left(V_{B}\right)_{i}=2 \pi r \cdot \frac{\pi\left(\lambda_{i} / 2\right)^{2}}{4}
$$

According to Eqs. (21) and (22), the mass breakup rate per unit area $\dot{m}_{B}$ may be computed by averaging the sum of the total breakup mass at each numerical marching step along the jet path:

$$
\dot{m}_{B}=\frac{\sum_{i=1}^{\infty} \rho\left(V_{B}\right)_{i}}{A_{\xi} \cdot \sum_{i=1}^{\infty}\left(t_{B}\right)_{i}}
$$

where $A_{\xi}$ is the jet surface area of each marching step (i.e., $A_{\xi}=2 \pi r \cdot \Delta \xi$ )

\section{JET BREAKUP AND ATOMIZATION}

When the relative velocity between liquid and gas phases for the Rayleigh jets [24] increases significantly, the effects of aerodynamic forces should be considered along with the effects of capillary forces. Consequently, the wind-induced regimes occur and the relative velocity becomes a controlling parameter. That is why Weber's analysis [27] predicts a continual increase in breakup distance with no peak at all with the increase of the exit velocity. This is because it does not take into account the effects of the ambient velocity upon the stability process. In the atomization regime, with further increase in relative velocity, the primary breakup mainly due to aerodynamic effects occurs around the jet surface and commences near the nozzle exit. As shown in Figure 2, the spray takes the form of a cone and breakup drop size becomes much smaller than other regimes. In the present study, the main interest was in the case of high-speed jets in the atomization regime. Therefore, only cases in the atomization regime were tested and discussed in this section. A water jet of diameter $D=2 \mathrm{~cm}$ injected at $u_{0}=1 \mathrm{~m} / \mathrm{s}$ into the air 
stream was considered as the first problem. Three constant air stream velocities $\left(u_{g}=50,100\right.$, and $200 \mathrm{~m} /$ s) were used for the present sensitivity study.

\section{Liquid jet shape and acceleration}

Figures 3 and 4 present predicted liquid jet-core shapes and their axial velocity distributions for all three gas-phase velocities. The predicted jet-core shapes in Figure 3 show that the highest relative velocity case $\left(u_{g}\right.$ $=200 \mathrm{~m} / \mathrm{s}$ ) gives the longest breakup length and the smallest jet diameter. The intact-core length increases with the gas stream velocity. It can be seen that in the case of the highest air stream velocity, the jet accelerates and elongates faster than in the other two cases. Figure 4 shows that with the increase in relative velocity, the jet is accelerated faster.

\section{Liquid breakup properties}

The present atomization model is capable of predicting the local jet breakup rate and droplet size distribution. Figure 5 presents predicted minimum and maximum droplet size range along the jet length for three gas-phase velocities. Several observations can be made from the drop size distribution through Figure 5. For larger air velocities, smaller drops are generated. The drop size range continuously increases with the jet streamwise distance for all three cases. The jet surface starts to breakup immediately at the nozzle exit. This means that the intact surface length in all three cases is very small. In the present computations, the flow conditions, $\mathrm{Re}=10^{6}$ and $\mathrm{Oh}=10^{-3}$, indicate that the breakup process occurs in the second wind-induced or atomization regime. In these regimes the jet intact

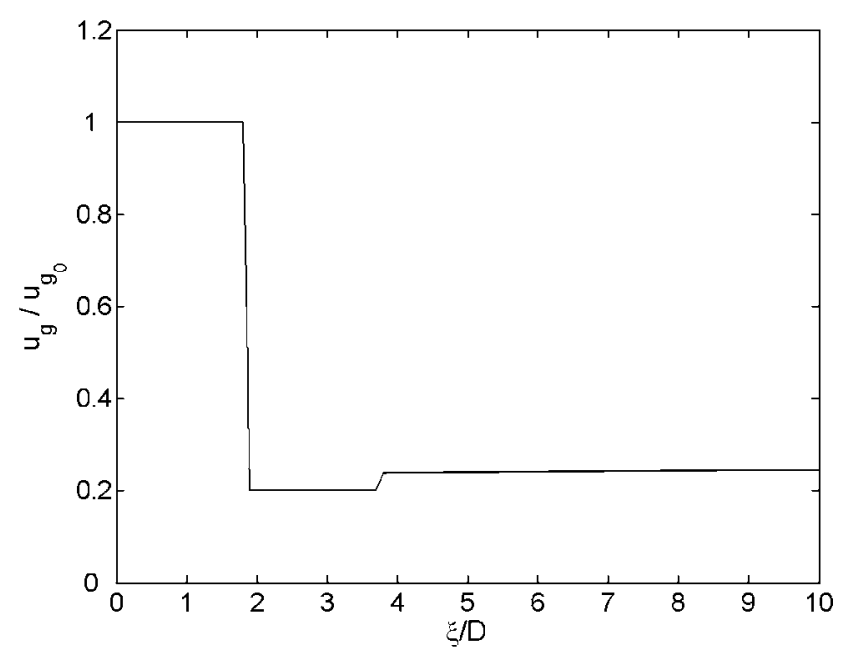

Fig. 3. Predicted shapes of a water jet into constant-speed air streams. surface length should be close to zero, which is well predicted by the present model.

The predicted surface breakup rate increases as the relative velocity increases, as shown in Figure 6. The slope of each curve is the breakup rate based on the local jet conditions. Comparing the case of $u_{g}=200 \mathrm{~m} / \mathrm{s}$ to the cases of $u_{g}=50 \mathrm{~m} / \mathrm{s}$ and $u_{g}=100 \mathrm{~m} / \mathrm{s}$ in Figure 6 , it is seen that at $\xi / D=5$, about fifty percent of mass has stripped from the jet for the case of the highest gas velocity, and smaller percent breakup mass strips for other cases.

In the atomization regime, smaller drops often separate from the liquid jet surface due to the aerodynamic effects, whereas larger drops detach from the tail

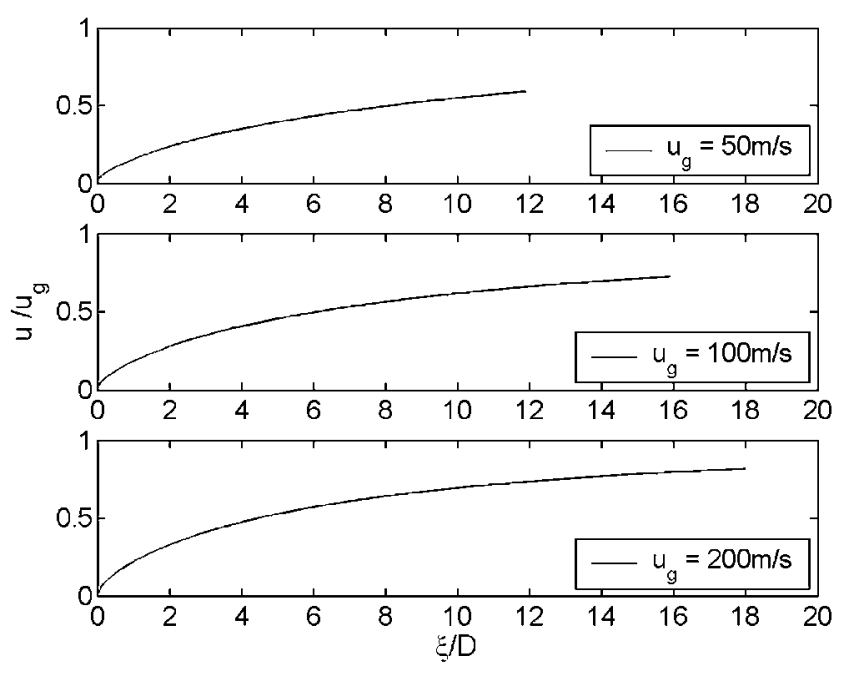

Fig. 4. Variations of axial velocity of a water jet into constant-speed air streams.

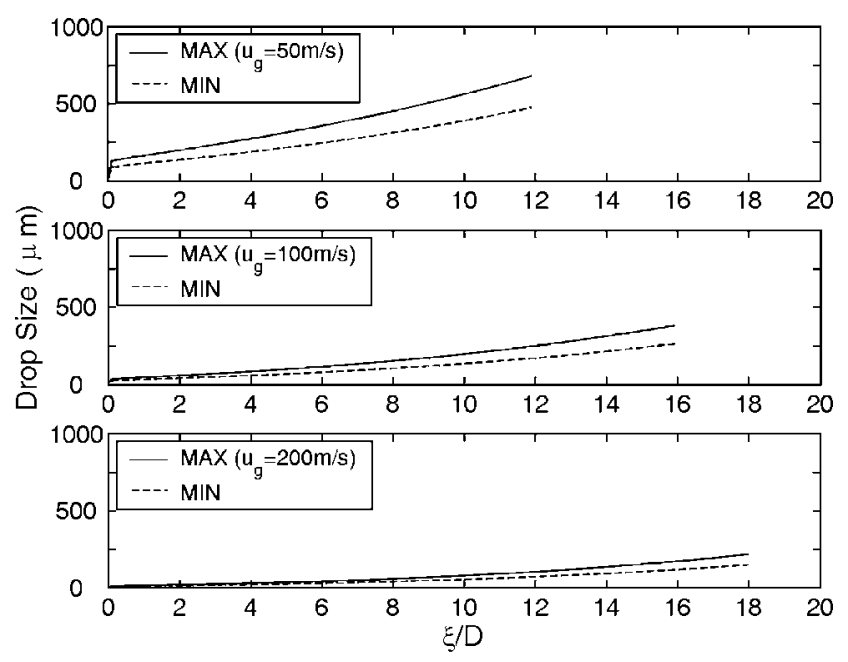

Fig. 5. Drop size distributions of a water jet into constant-speed air streams. 
of the jet due to the capillary effects. This phenomenon can be seen in Figures 5 and 6. In Figure 5, the drop size increases along the jet downstream distance and becomes the largest at the jet tail. At the tail section of the jet, large drops separate when the jet radius is of the size of the fastest growing wavelength. It is clear that the lower relative velocity case generates a larger drop size represented by the straight vertical section of the breakup curve of Figure 6.

\section{Comparison with the CICM}

Figure 7 presents the comparison between the breakup rates predicted by the present model and the semi-empirical formulae of the CICM [25]. The test case with gas velocity $u_{g}=100 \mathrm{~m} / \mathrm{s}$ is selected. Computations with the present model indicate that the spectral resolution (discrete wavelength $\Delta \lambda$ ) has an influence on the predicted breakup rate. Figure 7 presents the influence of the spectral resolution $\left(\Delta \lambda=10^{-6}, 10-7,10^{-9} \mathrm{~m}\right)$ on the predicted surface breakup rate.

The general form of the predicted breakup rate curve appears similar in all three cases, but oscillations occur close to the injector exit in the case of low resolution $\left(\Delta \lambda=10^{-6} \mathrm{~m}\right)$. Comparison of the present model with the CICM results, as shown in Figure 7, indicates that the predicted breakup rates are larger than the CICM. However, it is difficult to evaluate, and directly compare, both results since empirical constants of the CICM formula were correlated from experimental work of liquid propellant rather than water.

\section{DEMONSTRATION CALCULATIONS}

For the practical demonstration of the present
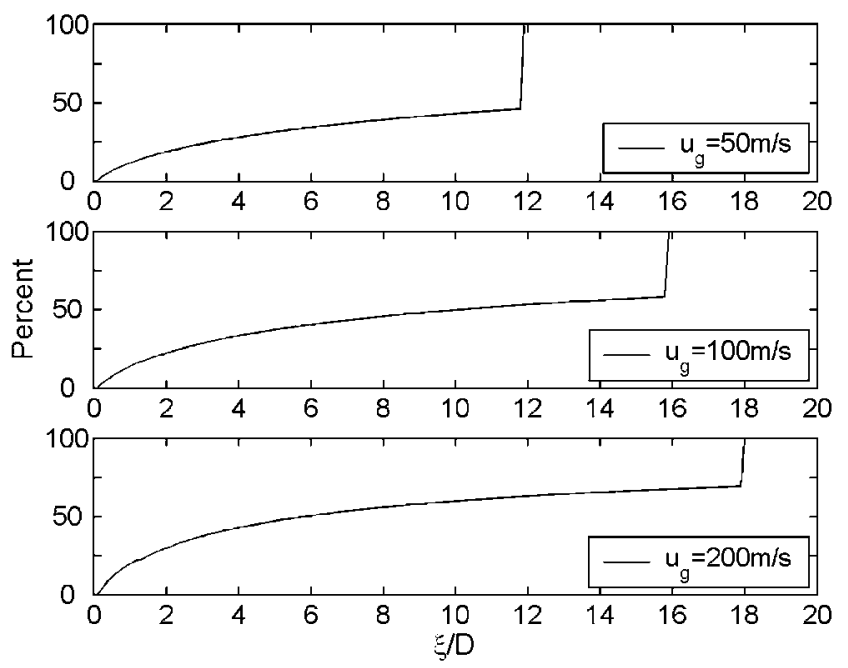

Fig. 6. Atomized mass percentages of a water jet into constant-speed air streams. model, the analysis of the single injector of the space shuttle main engine preburner was performed. A coaxial injector, with a liquid oxygen jet surrounded by an annular hydrogen gas stream, was considered. At the injector tip, typical velocities are $24.4 \mathrm{~m} / \mathrm{s}$ for liquid oxygen and $213 \mathrm{~m} / \mathrm{s}$ for gas hydrogen.

\section{Liquid jet shape and acceleration}

The calculated jet-core shape and the intact-core length are shown in Figure 8. The predicted intact-core length is about ten injector diameters. The radius decreases monotonically with the distance from the injector. Results of similar studies reported in the past $[13,14]$ indicate more non-uniform jet radius variation.

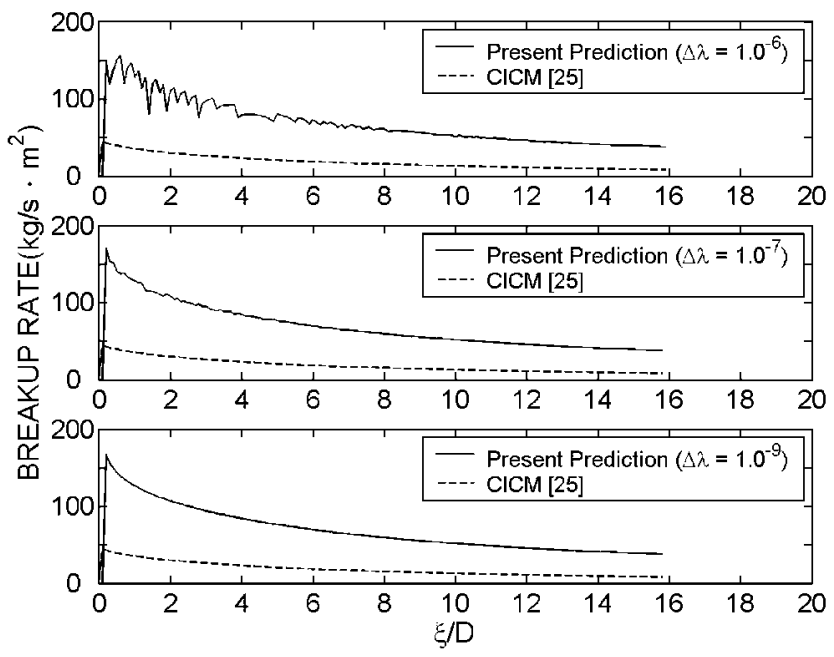

Fig. 7. Sensitivity analysis for primary breakup.

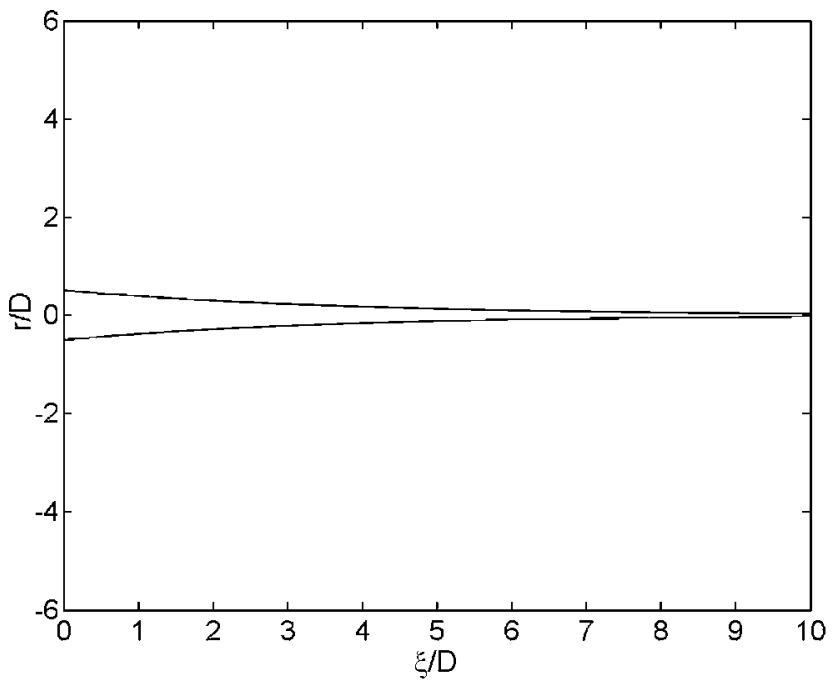

Fig. 8. Predicted shape of a LOX jet into a constant-speed $\mathrm{GH}_{2}$ stream. 
The difference can be attributed to the present assumption of non-reactive constant-velocity hydrogen gas flow, as well as direct coupling between atomization and gas-phase hydrodynamics is accounted for in the present computations.

Figure 9 presents the predicted liquid jet core velocity variation along the axial distance. A monotonic jet velocity increase up to $40 \%$ of the ambient gas velocity is observed.

\section{Jet breakup properties}

Figures 10 and 11 show the calculated primary breakup rate per unit area and drop size distribution

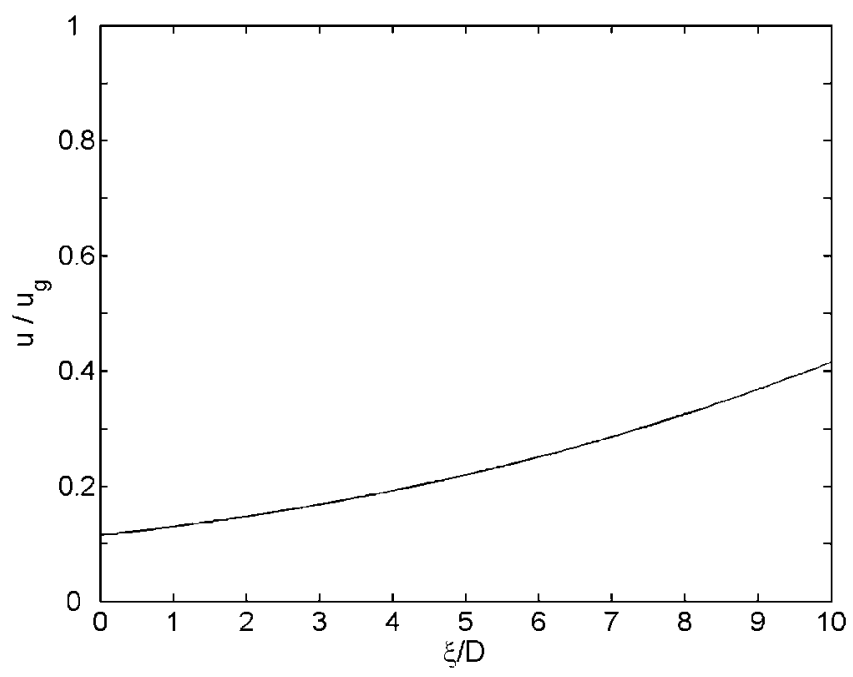

Fig. 9. Variation of axial velocity of a LOX jet into a constant-speed $\mathbf{G H}_{2}$ stream.

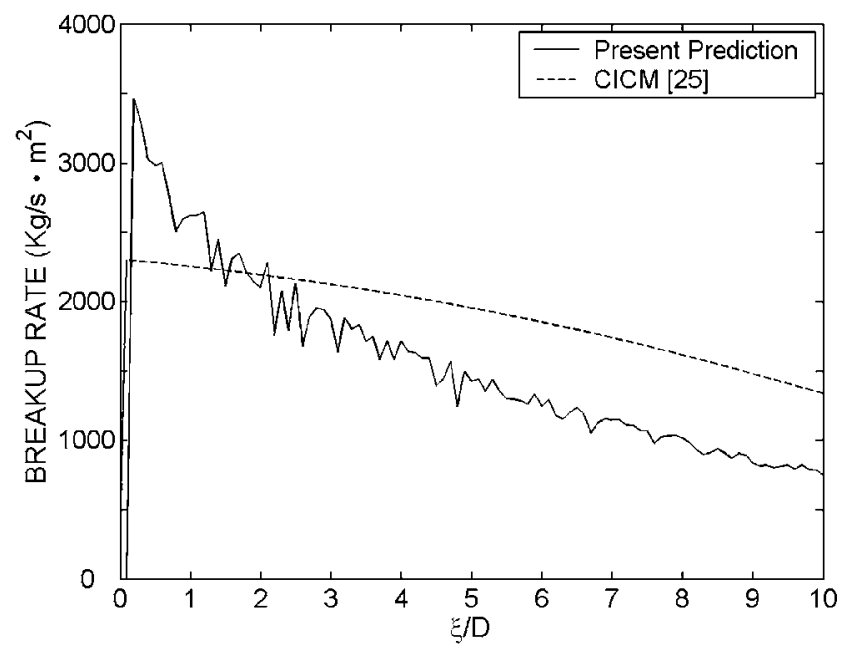

Fig. 10. Variations of primary breakup of a LOX jet into a constantspeed $\mathbf{G H}_{2}$ stream. with CICM results. In contrast to the water jet case, as shown in Figure 7, the comparison between present predictions and CICM results shows reasonably better agreement. This is probably due to the fact that CICM formulae have been directly calibrated for $\mathrm{LOX} / \mathrm{GH}_{2}$ flow. Again, the fluctuations along the curve are caused by the inadequate spectral resolution $\Delta \lambda$ as discussed in Figure 7.

Figure 12 shows the comparison of the present prediction with the CICM formula [25] and Liang's model [14] for atomized mass percentage of a LOX jet into a constant speed $\mathrm{GH}_{2}$ stream. Note that the results of Liang incorporate the semi-empirical formula of CICM in their atomization model. Present results show

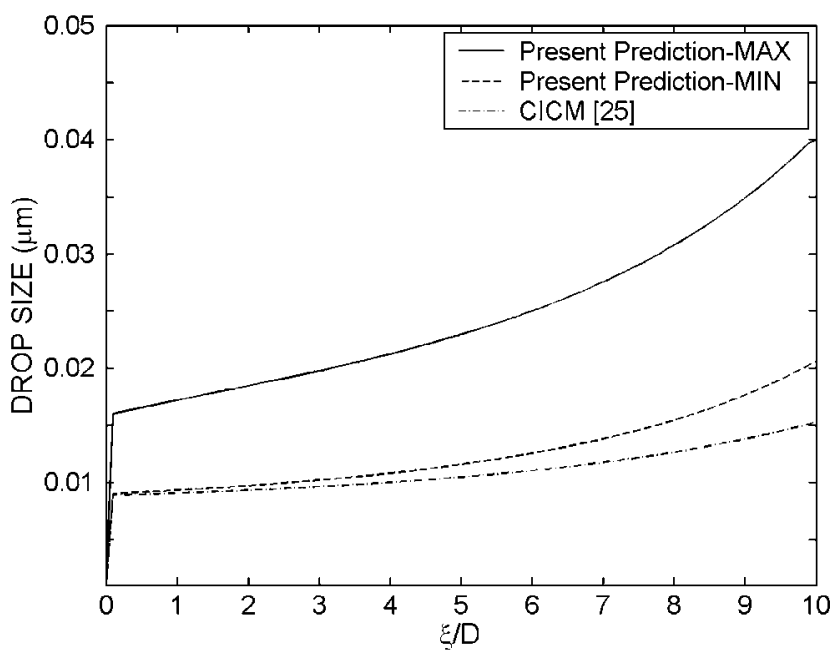

Fig. 11. Drop size distribution of a LOX jet into a constant-speed $\mathrm{GH}_{2}$ stream.

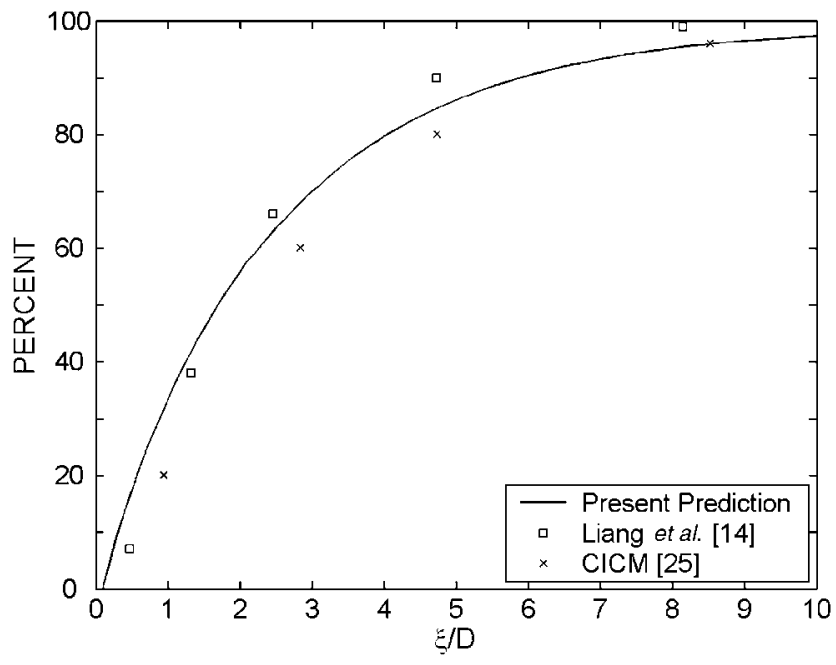

Fig. 12. Atomized mass percentages of a LOX jet into a constant-speed $\mathrm{GH}_{2}$ stream. 
slight over-prediction of the breakup rate along the jet length.

\section{Influence of gas aerodynamics}

The assumed constant gas velocity, used in the previous computations, does not adequately represent the complex reactive flow pattern in the vicinity of the injector. The gas expansion at the injector exit and consecutive acceleration at the ignition point are expected close to the SSME preburner injector exit.

Figure 13 presents postulated "step-varying" velocity profile in the injector cup and combustion regions of the preburner. The sudden velocity decrease in Figure 13 represents the hydrogen expansion within the cup region and small velocity increase in the downstream section represents the reaction front acceleration.

Figure 14 shows the comparison of the axial variation of the mean drop size predicted by Liang et al. [14] and by the present model with assumed "step-varying" velocity profile. A large increase in mean drop size should be noticed at the location of the velocity step, which is similar to the results of the recent study [7]. The predicted drop size in the combustor region, based on smaller relative velocity, agrees well with Liang's results, which utilize the semi-empirical formula of CICM. Note that the wave growth rate is strongly depended on the term of $u_{R}^{2}$ in Eq. (7) so that the present results for the drop size exhibits a step-varying distribution according to the velocity profile of Figure 13.

Figure 15 shows the comparison of the present prediction with the CICM formula [25] and Liang's model [14] for atomized mass percentage of a LOX jet into a $\mathrm{GH}_{2}$ stream with a "step-varying" velocity profile. From Figure 6, it is evident that the mass atomized at the jet upstream is faster than at the jet downstream due to

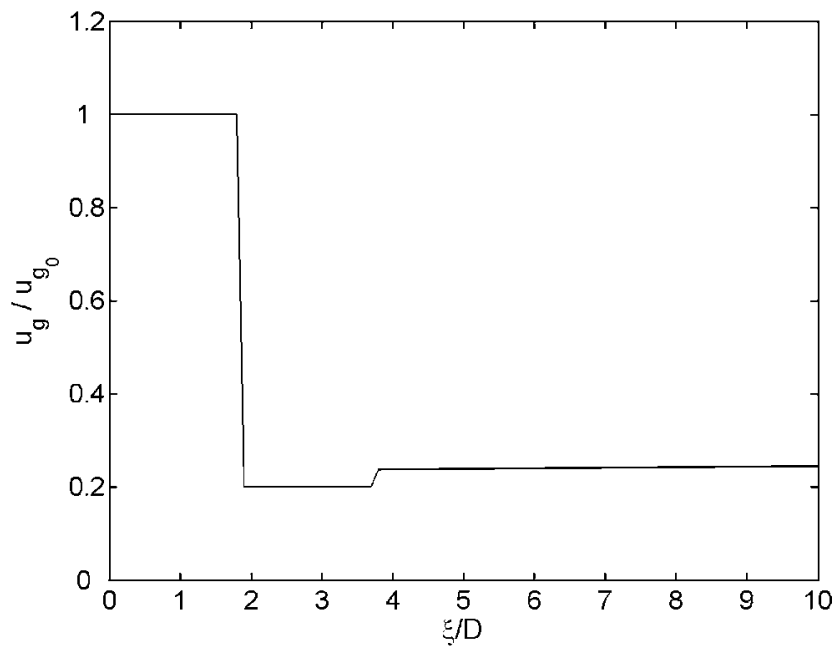

Fig. 13. Axial variation of hydrogen stream velocity using a step function. the maximum relative velocity at the nozzle exit and the jet-core acceleration along the downstream. This phenomenon can be also demonstrated in Figure 15. It is seen that about eighty percent of mass is stripped from the jet before $\xi / D=6$ for all models. Between $\xi / D=2$ and $\xi / D=6$, the effect of the hydrogen expansion slows down the stripping rate of the breakup mass atomized from the jet surface. As a result, the present prediction of atomized mass percentage of a LOX jet agrees with Liang's and CICM results. However, the present results show slightly underestimated breakup rate beyond $\xi / D$ $=6$. Accurate prediction of the atomization process would require the direct simulation of two-phase flows to account for the interactions between the jet breakup

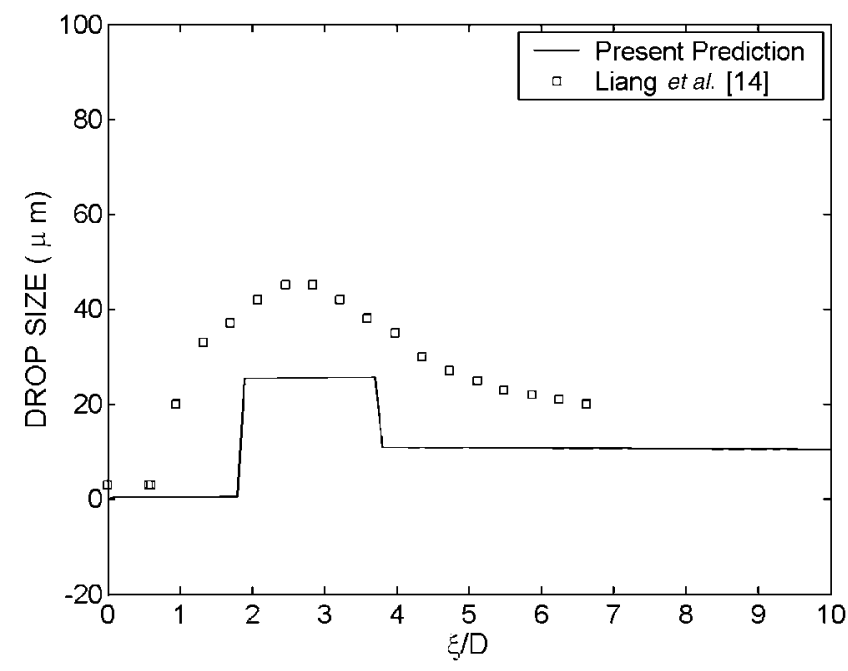

Fig. 14. Drop size distribution of a LOX jet into a non-constant-speed $\mathrm{GH}_{2}$ stream.

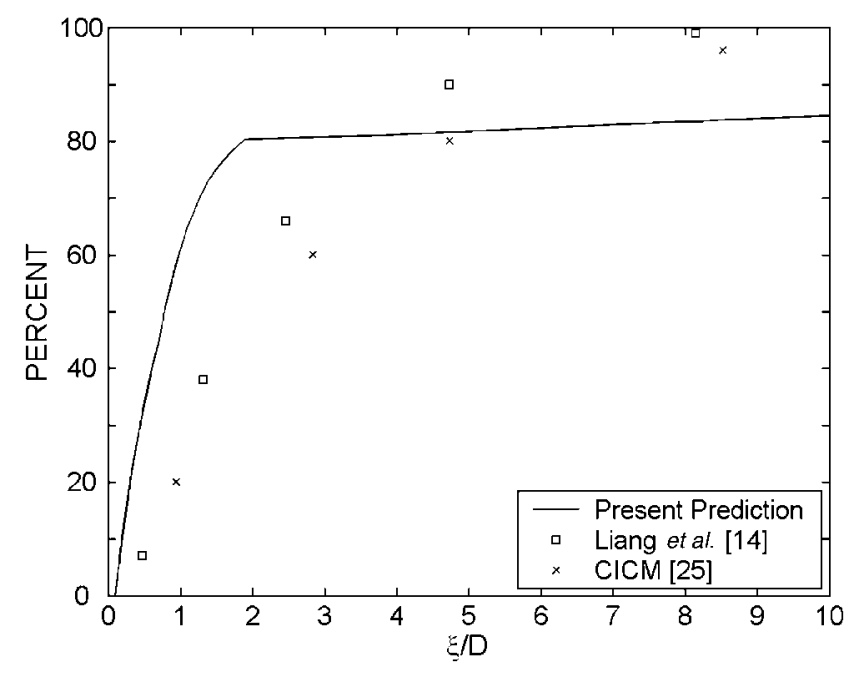

Fig. 15. Atomized mass percentages of a LOX jet into a non-constantspeed $\mathrm{GH}_{2}$ stream. 
and gas flow field.

\section{CONCLUSIONS}

In the present study, based on the wave instability analysis of the interface between liquid and gas, an atomization model for the high-speed liquid jets was established to predict the atomization of a coaxial injector in the liquid/gas engine combustor. The wave instability along the liquid jet surface was analyzed by numerically solving the wave dispersion equation. The wave growth rates and the solutions of the liquid flow structure were coupled in the atomization model to predict drop size distribution and breakup rate.

The liquid jet solutions show that the highest relative velocity case gives the longest breakup length and the smallest jet diameter. The intact-core length increases with the gas stream velocity. In the case of the highest air stream velocity, the liquid jet accelerates and elongates faster than in the other cases of lower gas velocity. For the flow conditions in the atomization regime, the liquid jet breakup should occur immediately at the nozzle exit. As a result, the jet intact surface length appears close to zero, and that is well predicted by the present model. In the atomization regime, smaller drops often separate from the liquid jet surface due to the aerodynamic effects, whereas larger drops detach from the tail of the jet due to the capillary effects. This phenomenon can be also predicted in the present computations.

For larger air velocities, smaller drops are generated and the drop size range continuously increases with the jet streamwise distance. The present calculated primary breakup rate per unit area and drop size distribution agree well with the results of the CICM code. The assumed constant gas velocity along the liquid/gas interface does not adequately represent the real flow pattern in the vicinity of the injector. The present result utilizing the sudden velocity due to the hydrogen expansion within the cup region confirms that the mass atomized at the jet upstream is faster than at the jet downstream. The comparison of the present prediction with the CICM formula [25] and Liang's model [14] for atomized mass percentage of a LOX jet into a nonconstant $\mathrm{GH}_{2}$ stream shows reasonably good agreement, and demonstrated the capability of the present atomization model.

\section{NOMENCLATURE}

$A_{\xi} \quad$ jet surface area of each marching step

$C_{f} \quad$ liquid/gas friction coefficient

$D \quad$ initial liquid jet diameter

I Bessel function of the first kind
$K$

Bessel function of the second kind

$m_{B} \quad$ liquid breakup rate per unit area

$\dot{m}_{B}^{\prime} \quad$ liquid breakup rate per unit volume

$n \quad$ disturbance wavenumber

$p \quad$ liquid pressure

Re Reynolds number

$r \quad$ liquid jet radius

$S_{c} \quad$ source term of continuity equation

$S_{m} \quad$ source term of momentum equation

$t \quad$ time

$t_{B} \quad$ breakup time

$u \quad$ axial velocity of liquid

$u_{0} \quad$ initial axial velocity of liquid

$u_{d} \quad$ axial velocity of liquid droplet

$u_{g} \quad$ axial velocity of gas

$u_{g 0} \quad$ axial velocity of gas

$u_{R} \quad$ liquid/gas relative velocity

$V \quad$ liquid velocity vector

$V_{B} \quad$ volume of breakup ligament

$v_{j} \quad$ velocity component in each coordinate direction

$\varepsilon \quad$ friction at liquid/gas interface

$\eta_{0} \quad$ initial disturbance amplitude

$\lambda$ disturbance wavelength

$\sigma \quad$ liquid surface tension coefficient

$\rho \quad$ liquid density

$\rho_{g} \quad$ gas density

$\xi \quad$ liquid jet path

$\tau_{i j} \quad$ liquid shear stress tensor

$v \quad$ liquid kinetic viscosity

$\omega$ disturbance frequency

$\omega_{r} \quad$ disturbance growth rate

\section{REFERENCES}

1. Adelberg, M., "Breakup Rate and Penetration of a Liquid Jet in a Gas Stream," AIAA J., Vol. 5, No. 8, pp. 14081415 (1967).

2. Adelberg, M., "Mean Drop Size Resulting from the Injection of a Liquid Jet Into a High-speed Gas Stream," AIAA J., Vol. 6, No. 6, pp. 1143-1147 (1968).

3. Char, J.M., Kuo, K.K., and Hsieh, K.C., "Observations of Break-Up Processes of Liquid Jets Using Real-Time X-Ray Radiography,” J. Prop. Power, Vol. 6, No. 5, pp. 544-551 (1990).

4. Chuech, S.G., Przekwas, A.J., and Singhal, A.K., "Numerical Modeling for Primary Atomization of Liquid Jets," J. Prop. Power, Vol. 7, No. 6, pp. 879-886 (1991).

5. Faeth, G.M., "Mixing, Transport and Combustion in Sprays," Progr. Energy Combust. Sci., Vol. 13, No. 2, pp. 293-345 (1987).

6. Hirt, C.W. and Nichols, B.D., "Volume of Fluid (VOF) Method for the Dynamics of Free Boundaries," J. Comp. Fluids, Vol. 39, No. 2, pp. 201-225 (1981). 
7. Inamura, T. and Daikoku, M., "Numerical Simulation of Droplet Formation from Coaxial Twin-Fluid Atomizer," Atom. Sprays, Vol. 12, pp. 247-266 (2002).

8. Jeffreys, H., "On the Formation of Water Waves by Wind," Proceedings of the Royal Society, The Royal Society, London, Vol. 107, pp. 189-205 (1925).

9. Lamb, H., Hydrodynamics, 6th ed., Dover, New York (1945).

10. Lefebvre, A.H., Gas Turbine Combustion, Hemisphere, New York, pp. 374-375 (1983).

11. Lefebvre, A.H., Atomization and Sprays, Hemisphere, New York (1989).

12. Levich, V.G., Physicochemical Hydrodynamics, Prentice Hall, Englewood Cliffs (1962).

13. Liang, P.Y., "Liquid Rocket Combustor Computer Code Development," Proceeding of High-pressure Oxygen/ Hydrogen Technology Conference, NASA CP-2372, pp. 696-716 (1984).

14. Liang, P.Y., Jensen, R.J., and Chang, Y.M., "Numerical Analysis of SSME Preburner Injector Atomization and Combustion Processes," J. Prop. Power, Vol. 3, No. 6, pp. 508-514 (1987).

15. Lin, S.P. and Reitz, R.D., "Drop and Spray Formation From a Liquid Jet," Annu. Rev. Fluid Mech., Vol. 30, pp. 85-105 (1998).

16. Mayer, E., "Theory of Liquid Atomization in Hugh Velocity Gas Streams," ARS J., Vol. 31, No. 12, pp. 1783-1785 (1961).

17. Mayer, W., Schik, A., Schaffler, M., and Tamura, H., "Injection and Mixing Processes in High-pressure Liquid Oxygen/Gaseous Hydrogen Rocket Combustors," J. Prop. Power, Vol. 16, No. 5, pp. 823-828 (2000).

18. Mayer, W., Schik, A., Vieille, B., Chaveau, C., Goekalp,
I., Talley, D., and Woodward, R., "Atomization and Breakup of Cryogenic Propellants under High-Pressure Subcritical and Supercritical Conditions," J. Prop. Power, Vol. 14, No. 5, pp. 835-842 (1998).

19. Przekwas, A.J., Chuech, S.G., and Gross, K.W., "Computational Modeling Development for Liquid Propellant Atomization," Proceedings of the 25th JANNAF Combustion Meeting, Vol. 11, NASA Marshall Space Flight Center, Huntsville, AL, pp. 81-92 (1988).

20. Reitz, R.D. and Bracco, F.V., "Mechanisms of Breakup of Round Liquid Jets," Encyclopedia of Fluid Mechanics 3, GULF, New Jersey, pp. 233-249 (1986).

21. Reitz, R.D., "Modeling Atomization Processes In HighPressure Vaporizing Sprays," Atom. Spray Tech., Vol. 3, pp. 309-337 (1987).

22. Sallam, K.A., Dai, Z., and Faeth, G.M., "Liquid Breakup at the Surface of Turbulent Round Liquid Jets," Int. J. Multiphase Flow, Vol. 28, pp. $427-779$ (2002).

23. Sirignano, W.A., "Fuel Droplet Vaporization and Spray Combustion Theory," Progr. Energy Combust. Sci., Vol. 9, No. 2, pp. 291-322 (1983).

24. Strutt, J.W. and Rayleigh, B., The Theory of Sound, 2nd Ed., Maemillan, Lonfon (1896) (reprinted in 1945, Dover, New York).

25. Sutton, R.D., Schuman, M.D., and Chadwick, W.D., "Operating Manual for Coaxial Injection Combustion Model," NASA CR-129031 (1974).

26. Watson, G.N., A Treatise on the Theory of Bessel Functions, 2nd Ed., Combridge University Press, Cambridge (1944).

27. Weber, C., "Zum Zerfall eines Flussigkeitsstrahles (On the Disruption of Liquid Jets)," Z. Angew, Math. Mech., Vol. 11, p. 136 (1931). 